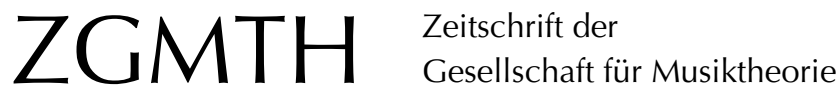

Neuwirth, Markus (2003/05): David Temperley, The Cognition of Basic Musical Structures, Cambridge, Mass.: The MIT Press 2001. ZGMTH 1-2/2/2-3, 304-319. https://doi.org/10.31751/196

(C) 2003/05 Markus Neuwirth

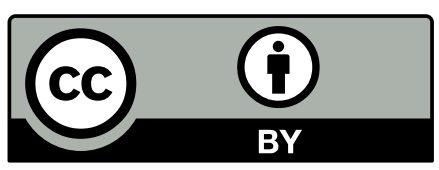

Dieser Text erscheint im Open Access und ist lizenziert unter einer Creative Commons Namensnennung 4.0 International Lizenz.

This is an open access article licensed under a Creative Commons Attribution 4.0 International License.

veröffentlicht / first published: 01/04/2005

zuletzt geändert / last updated: 01/12/2008 


\section{David Temperley, The Cognition of Basic Musical Structures, Cambridge, Mass.: The MIT Press 2001}

Die vorliegende Rezension beansprucht nicht, einenvollständigen Überblicküber dasgenannte Buch zu geben. Stattdessen verfolgt sie das Ziel, in Form von sechs Kurzessays Fragestellungen quer zur bestehenden Kapitelstruktur schlaglichtartig zu beleuchten. Der systematische Ansatz legt dabei die Berücksichtigung weiterer Arbeiten nahe: Es werden Aufsätze zur Sprache kommen, die David Temperley seit dem Erscheinen seines Buches in diversen Zeitschriften publiziert hat. Neben dem Facettenreichtum der musiktheoretischen Forschung Temperleys soll dadurch insbesondere der Weg zu seiner nächsten, für das Jahr 2006 geplanten selbständigen Veröffentlichung "Music and Probability" deutlich werden ein Weg, der durch einen Paradigmenwechsel vom sogenannten Präferenzregelansatz der Linguistik hin zur Wahrscheinlichkeitstheorie (in Form bayesianischer Modelle) gekennzeichnet ist. Um sowohl die Abhängigkeit von als auch den Kontrast zu anderen Ansätzen aufzuzeigen, sollen neben Temperleys eigenen Arbeiten weitere prominente Theorien in den Blick genommen werden.

Vorab ein kurzer Überblick über die Organisation und den Inhalt des Buches: Temperley gliedert seine Arbeit in zwei Teile. Der erste Teil stellt - nach einer Diskussion über den Status und die Ziele der Musiktheorie - den Präferenzregelansatz vor und wendet ihn auf sechs Bereiche der sogenannten musikalischen Basisstruktur an: Metrik, melodische und kontrapunktische Struktur, Tonhöhenbezeichnung und Repräsentation von Tonhöhenklassen, harmonische Struktur (Grundtonidentifizierung) und Tonartenstruktur (Tonartidentifizierung). Die Ausgangsfrage des ersten Teils lautet dabei - allgemein formuliert - wie folgt: Wie erschließen sich Hörer die einzelnen Komponenten der musika- lischen Basisstruktur aus den Informationen, die die musikalische Oberfläche anbietet? Im zweiten Teil, der mit »Extensions and Implications" überschrieben ist, werden Möglichkeiten diskutiert, wie der Präferenzregelansatz um die Aspekte der Revision, Ambiguität und Expektanz erweitert und auf Rockmusik und afrikanische Musik angewendet werden kann. Im Anschluß daran demonstriert Temperley das Potential des Präferenzregelansatzes für die Lösung von Problemen im Bereich musikalischer Komposition und Performance. Daneben wendet er sich auch Fragen musikalischer Stilistik und Bedeutung zu.

Das Repertoire, das Temperley schwerpunktmäßig untersucht, entstammt dem 18. und 19. Jahrhundert. Die Musik aus diesem Zeitraum - Temperley nennt sie "commonpractice music « oder »tonal music - zeichnet sich dadurch aus, daß sie dem enkulturierten Hörer, um dessen Wahrnehmungsvorgänge es hier geht, am geläufigsten ist.

Der Adressatenkreis, den Temperleys Buch ansprechen soll, ist relativ groß: Er umfaßt Musiker und Musiktheoretiker ebenso wie (Musik-)Psychologen, Musikinformatiker und KI-Forscher. Die verhältnismäßig einfache Sprache erleichtert die Lektüre ebenso wie die Tatsache, daß Temperley darum bemüht ist, kapitelübergreifende Zusammenhänge herzustellen.

1. Status, Ziele und Methoden der Musiktheorie

"We take the goal of a theory of music to be a formal description of the musical intuitions of a listener who is experienced in a musical idiom. « Mit dieser Positionierung der Musiktheorie im Bereich einer interdisziplinär aus- 
gerichteten Kognitionswissenschaft eröffneten Fred Lerdahl und Ray Jackendoff im Jahr 1983 ihre sowohl in musiktheoretischer wie auch -psychologischer Hinsicht einflußreiche Generative Theorie der tonalen Musik (GTTM). Knapp 20 Jahre später knüpft David Temperley, ehemals Doktorand bei Lerdahl, an diese Theorie an. Auch Temperley sieht die Aufgabe der Musiktheorie darin, die größtenteils automatischen (d.h. nicht-bewußt ablaufenden) kognitiven Verarbeitungsprozesse eines in einem bestimmten Kulturkreis serfahrenen Hörers im Rekurs auf musikalische Strukturen zu beschreiben und zu erklären. Dieses deskriptive Projekt der Musiktheorie unterscheidet er von einem präskriptiven Unternehmen. ${ }^{2}$ (Aufgrund der pejorativen Konnotation verwendet Temperley statt spräskriptiv den englischen Ausdruck "suggestive", der sich ins Deutsche kaum angemessen übersetzen läßt.)

Musiktheorie im präskriptiven Sinn zielt darauf $a b$, neue Arten des Musikhörens zu etablieren und gegebenenfalls das bestehende Hören zu bereichern. Diese Aussage impliziert, daß die von einer präskriptiven Musiktheorie beschriebenen Strukturen prinzipiell wahrnehmbar sein müssen, auch wenn es die Anleitung des Musiktheoretikers erfordert, daß sie tatsächlich wahrgenommen werden. ${ }^{3}$ Allerdings muß ausgeschlossen sein, daß die musikalischen Vorgänge und Strukturen, auf die sich die präskriptive Musiktheorie bezieht, von einem enkulturierten Normalhörer (s.u.) gegenwärtig wahrgenommen werden, auch wenn dies unbewußt geschehen sollte. Ansonsten würde sich der präskriptive Ansatz von einem deskriptiven nicht mehr unterscheiden.

Als ein prominenter Vertreter einer präskriptiven Musiktheorie ist Nicholas Cook zu nennen, der in einer empirischen Arbeit zur Wirkung tonaler Geschlossenheit die Auffassung vertritt, daß Musiktheorie, begreift man sie aus historischer Perspektive, von jeher dazu bestimmt war, didaktische Hilfestellung im Hinblick auf das Komponieren von Musik zu leisten (Musiktheorie als Kompositionslehre). ${ }^{4}$ Musiktheorie ist diesem Verständnis nach eine pädagogisch motivierte Handwerkslehre und fällt demnach in den Bereich der 'Poiesis`, die im Sinne der antiken, genauer: aristotelischen Klassifizierung Praxis und eben nicht Theorie ist. ${ }^{5}$ Heutige Musiktheorien (wie die Theorie Schenkers oder die GTTM) haben Cook (1987) zufolge die Aufgabe, die konzeptuellen Vorbedingungen bzw. die Prinzipien, die den Schaffensprozeß von Komponisten des 18. und 19. Jahrhunderts bestimmt haben, explizit zu machen. Bestenfalls - so Cook sei Musiktheorie dazu in der Lage, das Hören zu bereichern. Cooks Auffassung steht dabei im Kontext einer Immunisierungsstrategie, die darauf abzielt, musiktheoretische Aussagen vor der Falsifizierung durch empirisch gewonnene Erkenntnisse zu bewahren.

In späteren Arbeiten stellt Cook die Bedeutung der Musiktheorie für die Wahrnehmung stärker heraus: » [...] the purpose of analysis is not to reflect how people listen to music, but to explain how they ought to. Such was Schenker's position. « ${ }^{6}$ An anderer Stelle verschärft Cook seine These: »The aim of music theory, then, is to go beyond perception. [...] from the music-theoretical point of view, an analysis can value only to the extent that it deviates from perception. $\aleph^{7}$ In Music, Imagination, and Culture (1990) vertritt Cook die Auffassung, daß Musiktheorie eine Art metaphorisches oder imaginatives Hören ermöglicht. ${ }^{8}$ Hören im Sinne des theoriegeleiteten 'musicological listening، ist dabei eine Sache der Intention: „The whole question of deciding 'what one hears is problematical. After all, I can shear the most preposterous relationships if I choose to; it is a question of deciding [kursiv: M.N.] what I want to hear. ${ }^{9}$ Diese Aussage impliziert, daß der Hörer zwischen verschiedenen Auffassungen der Musik willentlich hin und her wechseln kann und dadurch in der Lage ist, die natürlichen Beschränkungen der Wahrnehmung hinter sich zu lassen.

David Temperley wirft Cook vor, nicht exakt zwischen den beiden oben genannten Theorietypen zu unterscheiden. Er zitiert eine Äußerung Cooks, die sich problemlos als 
Darlegung des deskriptiven Programms lesen läßt: "What we want an analysis for is to explain the powerful sense of cohesiveness and direction that pervades the discontinuities of the musical surface; and this is precisely what Schenker's sketch does. In the same way, we do not need Schenkerian analysis to tell us that there is a break at m. $16^{10}$; we need it in order to understand why this break seems so curiously evanescent, with the musical motion continuing after it as if nothing had happened [kursiv; M.N.]. «11 Diese Aussage legt den Schluß nahe, es gehe in der Analyse darum, einen phänomenal gegebenen, allgemeinen Höreindruck im Rekurs auf Strukturen des Notentextes zu erklären. So erklärt ${ }^{12}$ Cook den unspezifischen Eindruck des Zusammenhangs und der Zielgerichtetheit, den besagte Passage trotz der Diskontinuitäten an der musikalischen Oberfläche aufweist, indem er auf die Divergenz zwischen Oberflächenmuster und zugrunde liegender Struktur - auf das spezifische Verhältnis zwischen Vorder- und Hintergrund - verweist. Ein derartiger Eindruck ist allerdings das Resultat multipler Informationsverarbeitungsprozesse und damit auch das Ergebnis des tatsächlichen Hörens. Obwohl dieser Eindruck und noch mehr dessen Ursachen schwer verbalisierbar sind, kann ein Hörer auf Nachfrage hin Aussagen über sein Hören präzisieren. Die Aufgabe der deskriptiven Musiktheorie bzw. -analyse bestünde Temperley zufolge darin, die Faktoren zu benennen, die für den genannten Eindruck kausal verantwortlich sind. ${ }^{13}$

Hören bzw. Hörbarkeit als Kriterium zur Unterscheidung der beiden Theorietypen ist dem Einwand ausgesetzt, daß - um mit Carl Dahlhaus zu sprechen - »auch abstruse Konstruktionen auditiv lernbar ${ }^{14}$ sind. Doch selbst wenn Hörer nach einer längeren theoretischen Ausbildung in der Lage sein sollten, etwa bestimmte Konzepte der spitch class set theory، (z. B. ız-related sets ) in einem Musikstück zu identifizieren, wäre es Temperley zufolge dennoch nicht angemessen, diese Theorie als deskriptiv zu bezeichnen. Deskriptive Musiktheorie befaßt sich mit (bevorzugt un- bewußten) musikbezogenen Verarbeitungsvorgängen von Hörern, die eben keine musiktheoretische Ausbildung absolviert haben. Allerdings muß darauf hingewiesen werden, daß die Grenzen zwischen den beiden Theorietypen nicht trennscharf zu ziehen sind; vielmehr ist von graduellen Übergängen auszugehen. Insbesondere muß man sich vergegenwärtigen, daß auch basale musikbezogene Verarbeitungsprozesse durch eine Art simplizite Theorie gesteuert werden. Es stellt sich also die Frage, worin die Differenzen zwischen dieser simpliziten Theorie oben beschriebenen internalisierten Theorie bestehen. Der alternative Versuch, präskriptive von deskriptiver Musiktheorie mit dem Verweis auf die spezifischen Untersuchungsgegenstände zu unterscheiden - erstere befasse sich mit komplexen musikalischen Sachverhalten, während sich zweitere auf Basisstrukturen der Musik konzentriert -, scheitert daran, daß dadurch Temperleys Theorie, die einerseits (nach eigenem Bekunden) deskriptiv ist, und sich andererseits - im zweiten Teil seines Buches - mit komplexeren Sachverhalten befaßt, nicht berücksichtigt werden könnte. Das wäre aber einzufordern, da Temperleys metatheoretische Diskussion (ebenso wie seine Musiktheorie), so legen es die zahlreichen Referenzen auf Musiktheorien unterschiedlicher Provenienz nahe, im wesentlichen keinen normativen, sondern einen deskriptiven Anspruch erhebt: Sie versucht die Frage zu klären, worin die Ziele faktisch existierender Musiktheorien bestehen.

Als Erweiterung der von Temperley vorgeschlagenen dichotomen Klassifizierung ließe sich die Möglichkeit von Musiktheorie bzw. Musikanalyse ${ }^{15}$ als >Artikulation der individuellen, präanalytischen Hörerfahrung eines Theoretikers anführen, wie sie Mark DeBellis (2002) diskutiert. Temperley selbst nennt noch zwei weitere, seiner Ansicht nach nachrangige Ziele, die Musiktheorie bzw. -analyse verfolgen kann: 1. Musikanalyse als Studium der objektiv gegebenen Textform von Musik, der Partitur. Musikanalyse hat diesem Verständnis nach nichts mit Wahrnehmung zu tun. Alter- 
nativ dazu: 2. Musikanalyse sieht ihre Aufgabe in der Erschließung der in einem Werk enthaltenen Intention eines Komponisten, ein Anspruch, von dem Temperley glaubt, er werde in heutigen Analysen kaum mehr vertreten.

Temperley gesteht zu, daß es selten möglich ist, existierende Musiktheorien eindeutig einem bestimmten Theorietypus zuzuordnen. Vielmehr nehmen diese, wie z. B. die Theorie Heinrich Schenkers, eine Zwischenstellung ein. Allerdings, so die vehemente Forderung Temperleys, sollte Klarheit darüber bestehen, welche Anteile einer Theorie welchem Theorietypus entsprechen.

Im Zentrum der Musiktheorie Temperleys steht der (psychologisch) deskriptive Ansatz, der im folgenden genauer charakterisiert werden soll. Die Methode der deskriptiven Musiktheorie ist im wesentlichen introspektiv, d.h. der Theoretiker prüft seine eigenen Intuitionen im Hinblick auf bestimmte strukturelle Aspekte der Musik und hofft darauf, daß die dadurch gewonnenen Erkenntnisse konsensfähig sind. Bildet eine große Anzahl von Hörern/Musiktheoretikern andere Intuitionen aus, so ist eine Falsifizierung musiktheoretischer Aussagen möglich. Introspektion war als wissenschaftliche Methode in der Psychologie stets dem Verdacht ausgesetzt, nicht vorurteilsfrei, sondern vielmehr theoriegeleitet zu sein. Dabei besteht prinzipiell die Gefahr der Veränderung und Beeinflussung des Untersuchungsgegenstandes durch Wissen, Erwartung und Erkenntnisinteresse. Diesem Einwand begegnet Temperley mit dem Verweis auf die introspektive Vorgehensweise eines Linguisten, der ebenfalls seine Intuitionen überprüft, in diesem Fall die Intuitionen bezüglich der Wohlgeformtheit und der grammatikalischen Korrektheit von Sätzen, ein Verfahren, das nicht dem genannten Verdacht unterliegt.

Zudem muß auf das (scheinbare) Paradox hingewiesen werden, daß es doch um unbewußt ablaufende Verarbeitungsprozesse geht, die durch Introspektion bewußt gemacht werden sollen. Gegen diesen Einwand führt Temperley - neben dem erneuten Verweis auf die Linguistik - das Argument ins Feld, daß be- stimmte, bewußt zugängliche mentale Zustände als Indikatoren für unbewußt ablaufende Verarbeitungsprozesse interpretiert werden können. Als Ergänzung zu einem introspektionistischen Ansatz sieht er den experimentellen Ansatz der kognitiven Musikpsychologie, die Hypothesen über unbewußte Verarbeitungsprozesse dadurch überprüft, daß sie mittels Instruktion von ihren Probanden in einem bestimmten Kontext (Stimulus) ein bestimmtes Verhalten fordert, das wiederum nicht ohne Rekurs auf (hypothetische) automatische Verarbeitungsprozesse erklärt werden kann. Temperley referiert und diskutiert im Verlauf seines Buches eine Reihe experimenteller Befunde, ohne allerdings in Erwägung zu ziehen, sich selbst auf das Feld empirischer Forschung zu begeben. Die Methode der Rekomposition, die Temperley am Ende seines Buches vorstellt (S. 349-354), kann als musiktheoretisches Äquivalent zur experimentellen Methode des Psychologen aufgefaßt werden. Ebenso wie diese steht sie im Dienste der Überprüfung von Hypothesen, über deren Wahrheitsgehalt bzw. Plausibilität zwar nicht eine Stichprobe von Hörern entscheidet, sondern die Intuitionen des Musiktheoretikers.

Neben der introspektiven und der experimentellen Methode tritt in Temperleys Buch insbesondere der computerwissenschaftliche Ansatz in den Vordergrund. Die Bedeutung dieses Ansatzes für das Verständnis menschlicher Informationsverarbeitungsprozesse liegt prinzipiell darin, daß im Fall einer erfolgreichen Simulation dieser Prozesse durch Computerprogramme Rückschlüsse über die zugrunde liegenden Regeln gezogen werden können.

Die Verbindung der introspektionistischen und der komputationalen Methode gestaltet sich bei Temperley wie folgt: Die mittels Introspektion enthüllten Intuitionen des Theoretikers werden in ein Regelwerk (s. 2.) überführt, das seinerseits in einen Algorithmus transformiert wird. Im nächsten Schritt erfolgt die Analyse eines Inputs (ein musikalischer Abschnitt in Form einer spiano-rolk-Repräsentation, siehe unten) im Hinblick auf die oben genannten musikalischen Basisstrukturen. Die 
Analyse wird von einem Computerprogramm auf der Grundlage des implementierten Regelwerks durchgeführt. Die Ergebnisse dieser Analyse werden in einem weiteren Schritt mit den intuitiven Einsichten des Theoretikers verglichen. Im Fall der Nichtübereinstimmung werden die getesteten Regeln modifiziert bzw. neue Regeln generiert. Gegen diese Vorgehensweise können zwei Argumente angeführt werden: Das erste Gegenargument verweist auf die Gefahr der Zirkularität, da die Einsichten des Theoretikers sowohl die Ausgangsbasis für die Formulierung von Regeln bilden wie auch die Instanz, an der die Korrektheit computergenerierter Analysen gemessen wird. Das zweite Gegenargument stützt sich auf die Tatsache, daß Temperley zur Demonstration seiner Theorie häufig sehr einfache Beispiele auswählt, da deren Analyse kaum zu Differenzen unter Musiktheoretikern führen sollte. Im Fall komplizierterer, ergo weniger konsensfähigerer Beispiele (wie z. B. der Exposition des 1. Satzes von Mozarts G-Dur-Streichquartett, KV 387, die Temperley unter dem Aspekt der Gruppierungsstruktur untersucht, siehe S. 7683) steht allerdings auch die Intuition des Theoretikers als Beurteilungsinstanz der Computeranalysen in Zweifel. Zudem stellt sich die prinzipielle Frage, weshalb den Intuitionen des Musiktheoretikers gegenüber den $>$ Erkenntnissen privilegierter Status eingeräumt werden sollte.

Welcher Input eignet sich für Temperleys dezidiert formuliertes Ziel, die Wahrnehmung musikalischer Basisstrukturen zu beschreiben? Eine Möglichkeit besteht darin, konventionelle Notate als Input zu verwenden. Diese enthalten allerdings Angaben über Metrik (via Takt), Tonhöhenbezeichnung und die Aufteilung der Einzelstimmen innerhalb der kontrapunktischen Textur etc. und sind in diesem Sinne bereits Interpretation. Sie liefern Informationen über Aspekte der Musik, die der Hörer ja erst noch erschließen soll. Aus diesem Grund fällt Temperleys Wahl auf sogenannte spianorolk-Repräsentationen, die sowohl über die Tonhöhen der einzelnen Noten als auch über deren Einsatz- und Endzeitpunkt (Angabe der onset- und offset-time in Millisekunden) informieren. Der Tonraum wird dabei als Kontinuum dargestellt, wodurch Informationen etwa über Tonraumnutzung oder Texturdichte unmittelbar aus der Graphik ablesbar sind - im Unterschied zu konventionellen Notentexten, die eine instrumentenspezifische Aufteilung der Einzelstimmen darstellen. `Pianorolk-Repräsentationen können automatisch aus MIDI-Dateien erzeugt werden, Dateien, die ihrerseits entweder auf konventionellen Notaten oder auf der Einspielung auf einem MIDI-Instrument beruhen. Im ersten Fall werden die einzelnen Notenwerte metronomisch exakt, im zweiten Fall agogisch differenziert wiedergegeben. Ein Vorteil von Temperleys Ansatz besteht darin, daß er beiden Input-Typen mehr oder weniger erfolgreich Rechnung tragen kann. Grundsätzlich kann man allerdings die kritische Frage stellen, ob ein derartiges Repräsentationssystem für Temperleys Ziel, menschliche Informationsverarbeitungsprozesse beim Musikhören zu untersuchen, überhaupt geeignet ist. Gibt es wirklich eine Entsprechung zwischen der graphischen Repräsentation von Tönen (in den Dimensionen Tonhöhe und -dauer) und mentalen Zuständen? Als Alternative hätte Temperley auch einen akustischen Input wählen können.

Der Hörertypus, dessen Verhalten Temperley zu erklären versucht, ist, wie bereits erwähnt, mit demjenigen der GTTM, auf die sich Temperley hier beruft, identisch. Es handelt sich um einen Hörer, der in einem bestimmten musikalischen Idiom/Stil Erfahrungen gesammelt hat und auf der Basis dieser Erfahrungen in der Lage ist, typische von untypischen kompositorischen Elementen zu unterscheiden sowie Fehler in der Aufführung von Musik zu erkennen. Es ist dabei nicht erforderlich, daß ein derartiger Hörer eine musikalische oder musiktheoretische Ausbildung absolviert hat. Die Aufgabe der Musiktheorie besteht darin, die musikalischen Intuitionen dieses Hörers zu beschreiben und wenn möglich zu formalisieren und in Regeln zu fassen. sIntuition im Sinne der GTTM meint nicht ein Wissen, über das ein Hörer verbal Aus- 
kunft geben könnte, sondern im Gegenteil ein nicht-bewußtes musikbezogenes 'Wissen Dieses Wissen ermöglicht dem Hörer die Organisation und Strukturierung der Informationen, die die musikalische Oberfläche anbietet. Insofern der Theoretiker die Intuitionen eines serfahrenen Hörers nen zu erfassen versucht, stellt er sich mit diesem auf die gleiche Stufe.

Lerdahl und Jackendoff geben zu, daß es sich bei ihrer Konzeption eines serfahrenen Hörers in mehrerlei Hinsicht um eine Idealisierung handelt, insbesondere deswegen, weil es - erstens - aufgrund der verschiedenartigen Vorerfahrungen mit Musik einen derartigen Hörer in der Realität nicht gibt und weil sie - zweitens - nur den finalen Zustand eines Informationsverarbeitungsprozesses beschreibt, der in der Realität Zeit benötigt und ständigen Revisionen unterzogen werden muß. Allerdings bestehen sie darauf, daß der heuristische Wert dieser Idealisierung anerkannt wird.

\section{Der Präferenzregelansatz}

Nicht nur das Konzept des serfahrenen Hörers`, sondern auch Temperleys Präferenzregelansatz geht auf die GTTM zurück. Die GTTM unterscheidet drei Typen von Regeln, die dazu dienen sollen, die Wahrnehmung von Musik zu beschreiben: 1. Regeln der Wohlgeformtheit (well-formedness rules). Sie bestimmen den Bereich prinzipiell möglicher (kognitiver) Analysen. 2. Präferenzregeln (preference rules). Sie haben die Funktion, aus dem Set möglicher Analysen diejenigen auszuwählen, die die Kriterien, die durch die Regeln spezifiziert werden, am besten erfüllen. Im Idealfall sollte einer Analyse am Ende eindeutig der Vorzug gegeben werden. 3. Transformationsregeln (transformational rules). Dieser Typus spielt in der GTTM nur eine untergeordnete Rolle und kommt bei Temperley nicht mehr vor.

Bei Temperley haben die Präferenzregeln neben ihrer selektiven Funktion die Aufgabe, bestimmte Analysen von Musik zu evaluieren und ihnen einen numerischen Wert zu- zuweisen. Die nach Maßgabe der einzelnen Präferenzregeln (innerhalb eines z.B. metrischen Systems) errechneten Punktwerte werden schließlich aufsummiert. Insofern ergeben sich quantitativ präzisierbare, graduelle Abstufungen der Eignung von Analysen innerhalb des Bezugsrahmens eines bestimmten, stilistisch vorgesehenen Präferenzregelsystems. (Temperley unterscheidet nicht, wie die GTTM, zwischen sschwachen ken Präferenzregeln, was insbesondere für den Fall eines Konflikts zwischen den einzelnen Präferenzregeln nützlich wäre.)

Der evaluative Aspekt von Präferenzregeln ermöglicht es Temperley, diese nicht nur zur Beschreibung von Wahrnehmungsvorgängen zu verwenden, sondern darüber hinaus auch für die Bestimmung objektiver Eigenschaften der Musik. Daß der bevorzugten Analyse eines Musikstückes unter Anwendung eines bestimmten Präferenzregelsystems ein bestimmter maximaler Punktwert zugewiesen werden kann, der geringer oder größer als der eines anderen Stückes ist, hängt eben von den Eigenschaften des Stückes selbst ab. Diese Argumentation erlaubt es Temperley, den Präferenzregelansatz für eine Theorie des musikalischen Stils zu nutzen (s. unter 4.).

\section{Metrische Struktur, Ambiguität, Revision und Expektanz}

Temperley eröffnet die Anwendung des Präferenzregelansatzes auf die Basisstrukturen der Musik mit einer Diskussion der metrischen Struktur. Die Ausgangsfrage lautet: Wie erschließen sich Hörer eine metrische Struktur aus den Informationen, die die musikalische Oberfläche anbietet? Die drei Präferenzregeln, auf die sich Temperley schwerpunktmäßig konzentriert, basieren auf denjenigen, die die GTTM vorgeschlagen hat. Als erste metrische Präferenzregel (MPR 1) nennt Temperley die sogenannte Ereignisregel: Präferiere eine Struktur, die betonte Schläge mit dem Einsatz möglichst vieler Ereignisse verbindet (Koinzidenz der Einsätze von Ereignissen). Dieser Re- 
gel fügt er die sogenannte Längen-Regel (MPR 2) hinzu: Präferiere eine Struktur, die betonte Schläge mit dem Einsatz von Ereignissen verbindet, die von langer Dauer sind. Dabei ist nicht die tatsächliche Dauer entscheidend, denn auch im Falle eines Staccato-Vortrags würde sich die metrische Struktur nicht verändern. Ausschlaggebend ist vielmehr das Zeitintervall zwischen dem Einsatz einer Note und dem der folgenden Note, das sogenannte IInteronset-Intervall (IOI), und zwar innerhalb eines bestimmten Tonhöhenbereichs ('registerbezogenes $\mid \mathrm{O} \ll)$, d. h. innerhalb einer Linie einer Textur. (Temperley bestimmt ohne nähere Begründung einen Wert von neun Halbtönen. $)^{16}$ Die dritte metrische Präferenzregel (MPR 3), die sogenannte Regularitätsregel, lautet: Präferiere auf jeder Ebene Schläge mit möglichst gleichen Abständen (Kriterium der Äquidistanz). Abweichungen sind innerhalb eines gewissen Toleranzbereiches erlaubt.

Neben den Faktoren Noteneinsätze, Notendauer und Regularität führt Temperley weitere Faktoren an, die für das Erschließen eines metrischen Musters von Bedeutung sind: Gruppierung, Harmonik, Lautstärke, Parallelismus und melodische Akzentstruktur. Dadurch zeigt sich bereits, daß eine Parametertrennung, wie sie Temperleys Vorgehensweise suggeriert, sachlich nicht gerechtfertigt ist. Um der Komplexität von Phänomenen wie Metrik, Harmonik oder Tonalität Rechnung tragen zu können, muß das Zusammenspiel aller Faktoren berücksichtigt werden. Doch dies führt leicht zu Schwierigkeiten bei der Programmierung der Computersoftware. Aus diesem Grund muß Temperley häufig bestimmte Einflußfaktoren von der Analyse ausschließen. Schon allein aufgrund dieser Beschränkungen des computerwissenschaftlichen Ansatzes leuchtet es also kaum ein, welchen Vorteil dieser für das Verständnis menschlicher Informationsverarbeitungsprozesse haben soll.

Insbesondere im Hinblick auf Metrik stellt sich die Frage, ob Metrik mit anderen musikalischen Basisstrukturen auf die gleiche Stufe gestellt werden kann, wie Temperley es tut. Welchen ontologischen Status hat Metrik? Jus- tin London vertritt in Hearing in Time (2004) die starke These, daß sich Metrik fundamental von anderen musikalischen Parametern wie Tonhöhe, Rhythmus, Timbre, Lautstärke etc. unterscheidet. ${ }^{17}$ Als Argument dafür kann die Tatsache angeführt werden, daß ein Physiker enorme Schwierigkeiten hätte, ein physikalisches Äquivalent zur Metrik zu benennen, wozu er im Falle der anderen Parameter in der Lage wäre, indem er auf bestimmte Eigenschaften akustischer Wellen rekurriert. Positiv gewendet läßt sich Londons These so formulieren, daß ein Metrum aus der Interaktion von Klangobjekt und Hörer entsteht. Der Hörer verwendet dabei zur Interpretation des musikalischen Inputs bestimmte metrische Schemata bzw. stellt bestimmte Hypothesen über ein Metrum auf, die durch den tatsächlichen musikalischen Verlauf falsifiziert werden können. London unterscheidet ferner zwei Phasen im Hinblick auf die Konstruktion eines Metrums: eine Rekognitionsphase, in der es um das Erkennen eines metrischen Musters geht (Objekteigenschaft), und eine daran anschließende Kontinuationsphase, in der ein bereits etabliertes metrisches Muster auf zukünftige Ereignisse projiziert wird, wodurch Antizipation und Erwartung möglich werden.

Durch die Verortung von Metrik im Hörer (Metrum als ein mentales Konstrukt) können darüber hinaus Phänomene wie z. B. das der llauten Pause`, wie sie im Falle des Höhepunkts der Durchführung im ersten Satz der Eroica (Takt 280) Musikgeschichte geschrieben hat, erklärt werden. Dies gilt ebenso für das Phänomen, daß ein und dieselbe Tonfolge metrisch anders interpretiert wird, je nachdem, welcher metrische Kontext zuvor etabliert bzw. welches metrische Schema aktiviert worden ist. Der Kontextsensitivität von Metrik kann Temperleys Ansatz kaum gerecht werden. Meiner Meinung nach stellt Londons Metrik-Theorie u.a. aufgrund seiner Berücksichtigung zyklischer Modelle eine brauchbare Alternative zu Temperleys Theorie dar.

Wie bereits angedeutet, kann der Präferenzregelansatz als ein post-hoc-Ansatz charakterisiert werden. Das bedeutet, daß er den 
finalen Zustand des musikbezogenen Informationsverarbeitungsprozesses beschreibt, nicht allerdings die temporale Struktur desselben. Aus diesem Grund kann er der prinzipiellen Vorläufigkeit und Revisionsbedürftigkeit jeder kognitiven Analyse von Musik nicht gerecht werden. Diese Beschränkung des Präferenzregelansatzes versucht Temperley im zweiten Teil seines Buches zu beseitigen.

Temperley unterscheidet dort zwei Arten von Ambiguität: die diachrone und die synchrone. Diachrone Ambiguität zeichnet sich dadurch aus, daß im Lichte neuer Ereignisse vorangegangene Ereignisse, die für sich genommen unzweideutig sind, reinterpretiert werden können. Ein Beispiel hierfür liefert der Beginn des dritten Satzes aus Beethovens Klaviersonate op. 14,2, den Temperley wie viele andere Notenbeispiele m. E. nur sehr unzureichend diskutiert: Temperley notiert über die ersten vier Takte des Notentextes zwei alternative metrische Interpretationen, wobei die eine ein 2er-, die andere ein 3er-Metrum vorschlägt. ${ }^{18}$ Gegen Temperleys Analyse können zwei Kritikpunkte vorgebracht werden: Erstens fehlt eine Begründung, weshalb ein Hörer die Hypothese 2er-Metrum nach fünfmaliger Bestätigung (Takt 1-2) aufgrund einer einmaligen Falsifizierung (Takt 3-4) in Frage stellen sollte. Zweitens fehlt eine Bemerkung darüber, innerhalb welcher zeitlichen Grenzen Ereignisse einer revidierten Analyse unterzogen werden können.

Im Gegensatzzur diachronen Ambiguitätist für die synchrone Ambiguität charakteristisch, daß in einem bestimmten Moment (simultan) zwei unterschiedliche, konfligierende Deutungen der Musik möglich sind. In der Sprache des Präferenzregelansatzes formuliert, zeichnet sich eine synchron ambige Stelle dadurch aus, daß die Präferenzregeln keiner der möglichen Analysen eindeutig den Vorzug geben.

Im Unterschied zum retrospektiven Modus der Revision ist Expektanz per definitionem prospektiv, d.h. zukunftsorientiert, obwohl die Genese von Erwartungen auf Vorerfahrungen beruht, die entweder aus der Kenntnis einer Menge von Stücken eines bestimm- ten Stils resultieren (Extra-opus), oder aus der aktiven Auseinandersetzung mit den Besonderheiten eines bestimmten Stückes selbst (Intra-opus), das an bestimmten Stellen mehr oder weniger spezifische, mehr oder weniger stark ausgeprägte Erwartungen über den Fortgang der Musik erzeugt. Die Bedeutung des Präferenzregelansatzes für das Verständnis musikalischer Erwartungen liegt darin, daß mit seiner Hilfe Vorhersagen über das Expektanzverhalten von Hörern getroffen werden können. Temperleys Ansicht zufolge erwarten Hörer, daß der Fortgang eines Musikstückes, das sich bereits als Exemplar einer bestimmten stilistischen Kategorie erwiesen hat, auch in der Folge kognitive Analysen mit einer stilspezifisch hohen Punktezahl (high-scoring analysis) ermöglicht. Temperley erläutert empirische Studien zum Thema 'Expektanzı, so z. B. das bekannte Experiment von Mark Schmuckler (1989); daneben findet sich auch eine knappe Äußerung zu Eugene Narmours Implication-Realization Model (1990), neben Leonard Meyers Emotion and Meaning in $\mathrm{Mu}$ sic (1956) das einschlägige Referenzwerk zum Thema musikbezogener Expektanz. Doch insgesamt hätte man sich eine differenziertere Darstellung des Themas erhofft, insbesondere im Hinblick auf den Nutzen des Präferenzregelansatzes für das Verständnis der Genese und Dynamik von Erwartungen. Die mangelnde Berücksichtigung neuerer Ansätze zu den einzelnen Themenbereichen, seien sie musiktheoretischer, musikpsychologischer oder computerwissenschaftlicher Provenienz, ist eine deutlicheSchwächevon Temperleys Buch.

4. Kontrapunktische Struktur, musikalischer Stil und Wahrscheinlichkeitstheorie

Metrik und melodische Phrasenstruktur, die beiden Bereiche, die Temperley zu Beginn seines Buches aus der Perspektive des Präferenzregelansatzes behandelt, entsprechen in der GTTM der sogenannten shythmischen Strukturı, die eine zZeitspannensegmentierung (stime-span segmentation`) ermöglichen 
soll. Durch sie werden die Zeitintervalle festgelegt, innerhalb deren Töne in ein Konkurrenzverhältnis treten, wenn es darum geht, die nächsthöhere hierarchische Ebene zu erreichen. Ein wesentliches Ziel der GTTM bestand darin, Schenkers Reduktionismus, dem häufig mangelnde Wissenschaftlichkeit vorgeworfen wurde ${ }^{19}$, durch ein rigides Regelsystem zu fundieren. Daran ist Temperley offensichtlich wenig interessiert. Schenker spielt in Temperleys Arbeit insgesamt eine marginale Rolle. Ebenso fehlen bei Temperley die für die GTTM so charakteristischen, von der Linguistik geborgten Baumdiagramme. Metrik und melodische Phrasenstruktur stehen bei Temperley also gleichberechtigt neben den anderen Basiskomponenten wie der harmonischen, kontrapunktischen oder tonalen Struktur.

Die Segmentierung des musikalischen Informationsflusses ist eine der wesentlichen kognitiven Aufgaben, die ein Hörer zu leisten hat. Dabei lassen sich zwei Arten von Segmentierung unterscheiden. Die horizontale Segmentierung, d.h. die Gliederung einer Linie innerhalb einer Textur, wird von Temperley unter dem Begriff der smelodischen Phrasenstruktur ${ }^{20}$ abgehandelt. Im Anschluß daran widmet er sich der vertikalen Segmentierung, deren Resultat eine kontrapunktische Struktur darstellt. Die Aufgabe des Hörers besteht dabei darin, die Töne der musikalischen Oberfläche so zu gruppieren, daß einzelne, klar unterscheidbare Linien und Stimmen innerhalb einer komplexen Textur entstehen können. ${ }^{21}$ Den Begriff der skontrapunktischen Struktur faßt Temperley dabei erstaunlich weit; es geht nicht um spezielle kontrapunktische Gattungen oder Kompositionstechniken, sondern allgemeiner um die simultane Verbindung multipler melodischer Linien. Ebenso ist der Begriff `Polyphonie in der Verwendungsweise Temperleys ein Synonym zu Mehrstimmigkeit, während >Monophonie` mit dem deutschen Ausdruck ,Einstimmigkeit zu übersetzen ist.

Nach welchen Regeln geschieht dieser Gruppierungsvorgang? Ebenso wie bei der melodischen Gruppierung wird bei der kontrapunktischen Gruppierung obligatorisch auf die Gestalttheorie Bezug genommen, insbesondere auf das Gesetz der Nähe, das in vielen Theorien zu diesem Thema eine prominente Rolle spielt, u. a. in dem von Temperley diskutierten computerwissenschaftlichen Ansatz von David Huron (1989, 2001). Das Gesetz der Nähe findet sich in einer Präferenzregel wieder, die mit Temperley wie folgt formuliert werden kann: Es werden bevorzugt diejenigen Töne in Stimmen integriert, die gewährleisten, daß die Intervalle innerhalb einer Stimme möglichst klein sind (Präferenz für kleine Intervalle innerhalb einer Stimme). Diese Regel beschreibt nicht nur die Wahrnehmung, sondern findet auch in der tatsächlichen Kompositionspraxis ihren Niederschlag. Insofern können mittels Präferenzregeln auch objektive Eigenschaften der Musik erfaßt werden (s. 2.). Auf diese Feststellung legt Temperley besonderen Wert, da es ihm auch darum geht, mit Hilfe des Präferenzregelansatzes einen Beitrag zum Verständnis musikalischer Stilistik zu leisten. 'Stilk beschreibt in einer ersten Annäherung die Gemeinsamkeiten von Musikstücken (Temperley, 2004). Dabei lassen sich ästhetische, intellektuelle (ideengeschichtliche), soziale und ökonomische Determinanten von Stil anführen. Temperley ist insbesondere an den musikinternen Determinanten des kompositorischen Schaffens interessiert. An dieser Stelle kommt der Begriff der Kommunikation ins Spiel. Daß ein kommunikativer Austausch erfolgreich ist, setzt im Falle der Musik ein wechselseitiges Verstehen zwischen Komponist, Interpret und Hörer voraus. Kommunikation läßt sich, auch wenn kreative Momente im Spiel sind, als regelgeleiteter Vorgang begreifen. ^Regek ist allerdings bei Temperley (2004) nicht mehr im Sinne einer Präferenzregel zu verstehen, sondern spezifiziert die Wahrscheinlichkeit, mit der eine bestimmte Struktur, die einer kommunizierten Nachricht entspricht, vorliegt, wenn eine bestimmte musikalische Oberfläche, das Medium der Kommunikation, gegeben ist. Die Verbindung von Kommunikationstheorie und Wahrscheinlichkeitstheorie (Informationstheorie) ist an sich kein neuer Gedanke. Blickt man zurück 
in die Geschichte der Musiktheorie, so stellt man fest, daß in den 50er und 60er Jahren vor allem im angelsächsischen Bereich (z. B. von Leonard Meyer ${ }^{22}$ ) versucht wurde, Musik als einen Prozeß zu begreifen, der von Übergangswahrscheinlichkeiten zwischen musikalischen Ereignissen bestimmt ist. Der Informationsgehalt einer musikalisch kommunizierten Nachricht hängt dieser Vorstellung zufolge von der Wahrscheinlichkeit bestimmter Ereignisse ab. Neu ist allerdings bei Temperley die Verwendung bayesianischer Wahrscheinlichkeitsmodelle anstelle der bislang prominenten Markoff-Ketten. Der Unterschied besteht darin, daß nicht die Auftretenswahrscheinlichkeit musikalischer Ereignisse unter Voraussetzung der vorangegangenen Ereignisse bestimmt wird, sondern die Wahrscheinlichkeit einer Struktur unter Vorgabe eines Oberflächenmusters. 'Struktur bezeichnet hier also die Lesart eines Oberflächenmusters. Die Radikalität, die gemeinhin mit dem Begriff des Paradigmenwechsels verbunden ist, wird bei Temperley dadurch abgemildert, daß er versucht, aus der Perspektive des bayesianischen Ansatzes eine neue Klassifizierung von Präferenzregeln - als istructure rules` vs. sstructureto-surface rules - einzuführen.

Im folgenden soll kurz erläutert werden, welche Implikationen Temperleys sogenannter `Communicative-pressure`-Ansatz für das Verständnis musikalischer Stilistik und kompositorischer Regeln enthält, insbesondere im Bereich der Stimmführungsregeln der klassischen Kontrapunktlehre. Temperley bezieht sich in diesem Zusammenhang auf David Huron (2001), der zur Beantwortung dieser Fragen einen maßgeblichen Beitrag geleistet hat. Die Stimmführungsregel, die das Verbot der Parallelführung vollkommener Intervalle (Quinte und Oktave) vorschreibt, ist - so Huron - nicht primär arbiträr, d. h. konventionell festgelegt, sondern vielmehr durch die (psychoakustischen) Gesetze der auditorischen Wahrnehmung motiviert. Da - erstens - vollkommene Konsonanzen dazu tendieren, als ein einzelner Ton wahrgenommen zu werden, und da - zweitens - Töne, die sich in denselben Intervallabständen fortbewegen (komodulieren), ebenfalls zur Verschmelzung tendieren, ist die Gefahr der Fusion vergrößert, wenn, wie im Fall der Parallelität vollkommener Konsonanzen, beide Merkmale gegeben sind. Ein zweites Beispiel liefert die ebenfalls als Verbot formulierte Regel der Vermeidung kleiner Intervalle in tiefen Lagen. Huron begründet dieses Verbot mit dem Verweis auf das psychoakustische Phänomen der auditorischen Maskierung, d. h. der Überlagerung (Interferenz) von Partialtönen zweier Töne, die sich in einem kritischen Intervallbereich befinden. Ein drittes bekanntes Beispiel ist die Vermeidung von Stimmkreuzungen, die dadurch motiviert ist, daß Menschen eher dazu tendieren, zwei Linien mit einer $\mathrm{V}$-förmigen Kontur (eine reguläre und eine umgekehrte $\mathrm{V}$-Form) zu hören, als eine $X$-förmige Bewegung, die durch zwei sich kreuzende Stimmen entsteht.

Besteht die Intention eines Komponisten darin, daß ein Hörer die musikalische Struktur, in diesem Zusammenhang also die verschiedenen Stimmen einer kontrapunktischen Textur, möglichst eindeutig wahrnehmen soll, so werden seine kompositorischen Entscheidungen von diesen perzeptuell motivierten Regeln nachhaltig beeinflußt. Allerdings darf man im Bereich der Kunst nicht den Fehler begehen und aus einer gegebenen (evtl. historischen) Kompositionspraxis Rückschlüsse auf die Hörgewohnheiten von Rezipienten schließen, denn es ist durchaus denkbar, daß Komponisten etwa aus ästhetischen Gründen die Entscheidung treffen, an bestimmten Stellen eines Stückes die Wahrnehmung unterschiedlicher Stimmen zu erschweren. Der Einfluß von Regeln, die in biologisch bedingten Beschränkungen der Wahrnehmung begründet sind, wird demnach immer noch durch die Ziele und Intentionen eines Komponisten vermittelt.

\section{Motivwahrnehmung, metrischer Parallelismus und Modularität}

Das Feststellen oder - weniger positivistisch formuliert - die Konstruktion eines Zusam- 
menhangs zwischen einzelnen Motivformen zählt zu den grundlegenden Handlungsweisen eines Musikanalytikers. Die entscheidende Instanz ist dabei zumeist das Auge, das visuell identische oder ähnliche Tonkonfigurationen aus dem musikalischen Notentext herausfiltert und vergleicht. ${ }^{23} \mathrm{Ob}$ und inwiefern diese motivischen Beziehungen sich auch einem auditiven Zugang erschließen, insbesondere der Wahrnehmung eines musiktheoretisch nicht vorgebildeten Hörers, ist nach wie vor eine unbeantwortete Frage. Zudem müßte geklärt werden, was damit gemeint sein soll, daß ein Hörer motivische Zusammenhänge wahrnimmt. Geschieht dies unmittelbar, unwillkürlich und ohne Aufwendung kognitiver Ressourcen, oder eher indirekt, im Modus des gezielten, bewußten Suchens, der auf (Vor-)Wissen beruht?

Diese Fragen hat sich Temperley bereits in einem Aufsatz zu »Motivic Perception and Modularity « ${ }^{24}$ gestellt. Allerdings ist der Motivbegriff, der seiner Diskussion zugrunde liegt, kaum konsensfähig. Angemessener wäre meiner Meinung nach anstelle von sMotiv der Begriff selementare Tonkonstellationen . In seinem Buch definiert Temperley den Ausdruck smotivische Strukturı als Netzwerk von Segmenten, die in einem Stück als ähnlich oder aufeinander bezogen wahrgenommen werden (»the network of segments in a piece heard as similar or related«, S. 326). Diese Formulierung suggeriert, daß die auditorische Wahrnehmung einer motivischen Beziehung konstitutiv ist für deren Existenz. Dies legt nahe, daß eine Motivstruktur so lange nicht besteht, wie sie nur von einem lesenden Analytiker erkannt wird.

Als wesentlichen Faktor für die Wahrnehmung von Motivbeziehungen nennt Temperley den sogenannten smetrischen Parallelismus`. Die Ähnlichkeit von Motivvarianten wird demnach schneller erkannt, wenn die strukturell bedeutsamen Töne an denselben Positionen innerhalb einer metrischen Struktur auftreten. Um den Effekt dieses Faktors zu demonstrieren, stellt Temperley - gemäß dem kontrafaktischen Prinzip der Methode der Rekompo- sition - eine Motivvariante in einen anderen metrischen Kontext. Der introspektive Befund erbringt, daß es nun schwer fällt, Ähnlichkeiten zwischen den Motivformen zu erkennen.

Der Begriff >Parallelismusı, dessen Definition der GTTM nach eigenem Zugeständnis einige Schwierigkeiten bereitet hat, impliziert dabei die unmittelbare Nachbarschaft der Motivvarianten. Die Wahrnehmung von Motivformen, die in einer größeren zeitlichen Distanz zueinander stehen, oder gar satzübergreifende Zusammenhänge etwa à la Rudolph Reti kann Temperley nicht erklären.

Worin besteht die kognitive Bedeutung motivischer Zusammenhänge? Mit dem Verweis auf musikpsychologische Experimente stellt Temperley insbesondere die Funktion einer motivischen Struktur für die Enkodierung und damit eine verbesserte Gedächtnisleistung heraus. Auch hier spielt die Metrik eine besondere Rolle, indem sie Repräsentationen von Motivformen auf unterschiedlichen Ebenen der metrischen Hierarchie erlaubt. Als weitere wesentliche Faktoren nennt Temperley die Ähnlichkeit des Tonhöhenverlaufs, insbesondere die Bedeutung des globalen Merkmals 'Konturı, sowie die rhythmische Identität. Daneben spielen auch Harmonik sowie tonale und kontrapunktische Struktur eine Rolle.

Temperleys Interesse gilt primär der direkten und automatischen Wahrnehmung einer motivischen Struktur, nicht dem von analytischem Wissen durchdrungenen Modus. Die Modularitätsannahme, eingebracht in den kognitionswissenschaftlichen Diskurs durch Jerry Fodor ${ }^{25}$ und prominent vertreten in heutigen musiktheoretischen Konzepten (GTTM, Narmour), soll erklären, warum manche Ähnlichkeitsbeziehungen automatisch entdeckt werden, während andere erst einer mühevollen mentalen Suche zugänglich sind. Diese Annahme basiert auf der Vorstellung, daß bestimmte Informationen in unterschiedlichen Systemen (Modulen) verarbeitet werden, die unabhängig voneinander operieren und enkapsuliert sind, d.h. keinen Informationsaustausch betreiben. Neben diesen Modulen existiert, so die Annahme, ein zentraler Ver- 
arbeitungsprozessor, der diese Informationen gegebenenfalls bündelt - ein im Kern cartesianischer Gedanke. In heutigen kognitionspsychologischen Ansätzen verliert diese Vorstellung immer mehr an Bedeutung zugunsten dezentral organisierter Netzwerkmodelle, die ohne die Annahme eines zentralen Prozessors auskommen. Aus wissenschaftshistorischer Sicht ist insbesondere die zeitliche Verzögerung, mit der neueste Modellvorstellungen aus der Kognitionswissenschaft die Musiktheorie erreichen, von Interesse.

\section{Musikalische Infrastruktur, Schema- theorie und die Bedeutung von Musik}

Nachdem Temperley zu Beginn des zweiten Teils den Präferenzregelansatz um die Aspekte der Ambiguität und insbesondere der prinzipiellen Revidierbarkeit von Analysen erweitert hat, kommt er auf die Implikationen dieses Ansatzes vor allem im Hinblick auf höherstufige musikalische Sachverhalte zu sprechen (Motivik, Stil, Komposition, Aufführung, Schemata, Spannung und Energie, Bedeutung von Musik).

Bereits zu Anfang des Buches versuchte Temperley, den Stellenwert musikalischer Basisstrukturen im Rekurs auf die Metapher der ımusikalischen Infrastrukturı zu erläutern. Den Begriff ,Infrastruktur definiert er wie folgt (S. 3): "As the term is commonly used, infrastructure refers to a network of basic structures and services in a society - largely related to transportation and communication - which are required for the society to function." Eine Infrastruktur zeichnet sich durch zwei wesentliche Merkmale aus: erstens durch ihre Allgegenwärtigkeit, zweitens durch ihren funktionalen Charakter, d.h. die Infrastruktur ist nicht Selbstzweck, sondern Mittel oder Bedingung für das Zustandekommen höherstufiger Zwecke. Übertragen auf den Bereich der Musik bedeutet das, daß Metrik, Harmonik, melodische und kontrapunktische Gruppierung etc., die in beinahe jedem Musikstück auf irgendeine Weise vorhanden sind, dazu beitragen, daß melodische Schemata, histo- risch gewachsene syntaktische und semantische Topoi und schließlich musikalische Bedeutung entstehen können. Im Gegensatz zu den Komponenten der Infrastruktur haben etwa melodische Schemata oder stilistische Topoi okkasionalen Charakter.

Ich möchte Temperleys Diskussion musikalischer Schemata herausgreifen, um sein Verständnis der funktionalen Bedeutung der Infrastruktur für die Konstitution höherstufiger musikalischer und musiktheoretischer Sachverhalte zu veranschaulichen.

Der Begriff des Schemas hat eine zweifache Bedeutung, eine subjektive und eine objektive. 'Schemar als kognitionspsychologischer Begriff bezeichnet ein mentales Modell, das die Verarbeitung und den Abruf von Informationen beeinflußt sowie die Generierung von Erwartungen ermöglicht. Andererseits bezeichnet 'Schemar eine objektive Gegebenheit, die einer Internalisierung als kognitives Modell vorausgehen kann. Die zweite Bedeutungsvariante ermöglicht die fruchtbare Anwendung des Schemabegriffs im Bereich der Stilanalyse, wie sie Robert Gjerdingen (1988) im Anschluß an Meyer (1973) und Narmour (1977) vorgeschlagen hat. Gjerdingen liefert in A Classical Turn of Phrase eine ausführliche Darstellung eines Wechselnoten-Schemas (`changing-note schema). Die Mitglieder dieser Schemaklasse müssen bestimmte typische Merkmalskombinationen aufweisen, um als Realisierung des Wechselnoten-Schemas gelten zu können. Dies ist eine attraktive und psychologisch realistischere Alternative zu der Vorstellung, daß eine starre Merkmalskombination, basierend auf der Unterscheidung zwischen notwendigen und hinreichenden Merkmalen, die Mitglieder eines Schemas integriert.

Temperley stellt die V-I-Kadenz (am Beispiel von Bachs französischer Suite Nr. 6 in E-Dur, BWV 817) als das Beispiel eines multipel realisierbaren Schemas vor. Sein Interesse gilt dabei nicht den kognitiven Konsequenzen eines Schemas, sondern dem Nachweis, daß die musikalischen Basiskomponenten einen unverzichtbaren Anteil an der Konstitution von Schemata haben. Als Beleg für seine The- 
se führt er Gjerdingens 1-7-4-3 Schema an. Er kommt letztendlich zu folgender Konklusion (S. 338): »In all these ways, then, infrastructural representations are necessary for perception of the 1-7-4-3 Schema [kursiv; M.N.].»

Dagegen kann eingewendet werden, daß diese Aussage entweder trivial ist in dem Sinne, daß selbstverständlich musikalische Basiskomponenten beteiligt sind, wenn bestimmte Schemata in der Musik erkannt werden, oder, daß sie den gängigen Schematheorien widerspricht, die eben nicht davon ausgehen, daß bestimmte Basiskomponenten notwendigerweise gegeben sein müssen, um ein Schema erfolgreich identifizieren zu können. Man kann sogar so weit gehen, die Schematheorie gegen Temperleys Beweisziel zu wenden: Schema als emergentes Phänomen bedarf gar nicht der vollständigen Verarbeitung aller Einzelkomponenten; die Identifizierung eines Schemas kann schließlich die Verarbeitung elementarer Eigenschaften in Richtung der aktivierten mentalen Repräsentation verzerren. In jedem Fall bleibt bei Temperley unklar, welchen Beitrag der Präferenzregelansatz für das Verständnis höherstufiger musiktheoretischer Entitäten leisten kann, eine Schwäche, die meiner Meinung nach den gesamten zweiten Teil charakterisiert.

In seiner Stellungnahme gegen die Arbitraritätshypothese ${ }^{26}$, die im Zusammenhang der Diskussion musikalischer Bedeutung ${ }^{27}$ zu verorten ist, kommt Temperley nochmals auf musikalische Schemata zu sprechen. Er versucht dabei die Hypothese zu widerlegen, daß die Bedeutung eines musikalischen Schemas arbiträr ist in bezug auf die infrastrukturellen Elemente, aus denen es besteht (Hypothese 3, S. 347-348). Die Frage, die Temperley in diesem Zusammenhang stellt, lautet: Ist die Tatsache, daß eine V-I-Kadenz Geschlossenheit impliziert, konventionell bestimmt, oder beruht sie auf allgemeineren Prinzipien wie dem Wechsel von Spannung und Stabilität? Die Tatsache, daß die Tonika als Folge der Dominante einen höheren Grad an Stabilität besitzt, wird dadurch bestätigt, daß innerhalb einer Tonart der Tonika ein höherer Punktwert zugewiesen wird.
Problematisch ist dabei, daß Temperley seine Fragestellung nicht allgemein diskutiert und Beispiele als zusätzliche Evidenz anführt, sondern sich sofort auf den Fall einer authentischen Kadenz konzentriert, was ihm die Widerlegung der Arbitraritätshypothese erleichtert. Die Diskussion anderer Schemata erscheint höchst zirkulär: »March music symbolizes the military because it is actually somewhat similar to military music." Damit die Aussage an Informationsgehalt gewinnen würde, müßte Temperley klären, worin die Ähnlichkeit zwischen Marschmusik und dem musikexternen Bereich ^Militär besteht. (Liegt die Ähnlichkeit in der Bewegungsqualität der Musik oder in deren Ausdruckscharakter?) Der Versuch, den Präferenzregelansatz auf höherstufige musikalische Konzepte anzuwenden, besteht bei Temperley mehr aus Andeutungen und Anregungen, nicht auf einer ausführlichen Diskussion. Positiv ist dabei allerdings, daß Temperley an dieser Stelle sowie das ganze Buch hindurch explizit auf die Schwächen seiner Arbeit verweist.

\section{Fazit}

Ziel der vorliegenden Rezension war es, in Form von Kurzessays bestimmte, meiner Meinung nach wesentliche Aspekte der Arbeit eines derzeit führenden Musiktheoretikers selektiv zu beleuchten, dies naturgemäß unter Ausblendung von Zusammenhängen, die beispielsweise in einer computerwissenschaftlich orientierten Zeitschrift mehr Beachtung gefunden hätten. Trotz der erwähnten Schwachstellen in Temperleys Buch sei noch einmal nachdrücklich betont, daß The Cognition of Basic Musical Structures das große Verdienst gebührt, der Musiktheorie erneut Möglichkeiten aufgezeigt zu haben, musikbezogene Verarbeitungsprozesse eines Normalhörers zu beschreiben und zu formalisieren. Vorbildhaft für die zukünftige Musiktheorie sollte insbesondere die Präzision sein, mit der Temperley musiktheoretische Aussagen formuliert und damit der Überprüfbarkeit zugänglich macht. 
Die Kritik an Temperleys Arbeit soll nicht die derzeit florierende Rezeption von Musiktheorie aus dem angelsächsischen Sprachraum blockieren; sie soll jedoch zu einem reflektierten Umgang mit diesen Arbeiten anregen. In jedem Fall wird man gespannt sein dürfen, in welche Richtung sich Temperleys wahr- scheinlichkeitstheoretisch inspirierter Ansatz entwickeln wird. Temperleys nächstes Buch Music and Probability(geplant für 2006) wird darauf eine Antwort geben.

\section{Markus Neuwirth}

\section{Anmerkungen}

1 Lerdahl / Jackendoff 1983, 1.

2 Eine im Vergleich zu Temperleys Buch ausführlichere Diskussion zu diesem Thema liefert Temperley 2001.

3 Siehe Temperley 2005, S. 94: „For a suggestive analysis to be good, it must be hearable, and the hearing that it brings about must be a rewarding and worthwhile one."

4 Cook 1987a, 204.

5 Es ist charakteristisch für Temperleys Diskussion des Theoriebegriffs wie für seine Theorie im allgemeinen, daß er eine historische Perspektive beinahe vollständig ausblendet.

6 Cook 1989, 123.

7 Cook 1994, 89.

8 Cook 1990. Z.B. S. 4: »A Schenkerian analysis is not a scientific explanation, but a metaphorical one; it is not an account of how people actually hear pieces of music, but a way of imaging them."

9 Cook 1987b, 57.

10 Cook bezieht sich auf den 1. Satz von Beethovens Klaviersonate op. 90.

11 Cook 1989, 132.

12 Der Begriff der `Erklärung` bedeutet in der Wissenschaftstheorie die Subsumierung eines Einzelfalls unter ein allgemeines Gesetz, das einen Kausalzusammenhang formuliert.

13 Für viele Mißverständnisse innerhalb der Musiktheorie oder auch zwischen Musiktheoretikern und Psychologen ist die Tatsache verantwortlich, daß kaum Einigkeit darüber besteht, was der Begriff ,Hören< bedeuten soll. Unter 'Hörenء können so unterschiedliche Phänomene subsummiert werden wie die Wahrnehmung von Ähnlichkeitsbeziehungen, das Identifizieren

eines metrischen oder kontrapunktischen Musters, die Wahrnehmung einer Passage als Rückleitung, das Hören eines Akkords als Spannungsauflösung oder als Dissonanz, oder schließlich das Hören eines Musikstücks als Fuge oder als Sonatenform.

14 Carl Dahlhaus 1987, 149.

15 Es führt zu einer gewissen Konfusion, daß Temperley die Begriffe `Theorie` und ^Analyse im Lauf seiner Argumentation beinahe austauschbar verwendet. Er bietet allerdings folgende Differenzierung an: Theorie unterscheidet sich von Analyse darin, daß sie sich mit der strukturellen Beschaffenheit von Musik im allgemeinen befaßt. Musikanalyse erprobt das von der Theorie zur Verfügung gestellte Instrumentarium an den Strukturen individueller Musikstücke. Dadurch wird eine Wechselwirkung von Theorie und Analyse möglich. Es stellt sich allerdings die Frage nach dem ontologischen Status der musikalischen Struktur: Befindet diese sich in der Textgestalt, im klingenden Objekt oder im 'Kopf des Hörers bzw. Theoretikers? Als Argument für die These, daß die Existenz musikalischer Strukturen im Wesentlichen auf Kognitionen höherer Ordnung (metaphorisches Denken) beruht, läßt sich Lawrence Zbikowskis Conceptualizing $\mathrm{Mu}$ sic (2002) lesen.

16 Die Bestimmung des rregisterbezogenen IOls« setzt bereits eine Analyse der kontrapunktischen Struktur voraus (s. Abschnitt 4) - ein weiteres Indiz für die Interdependenz der musikalischen Basisstrukturen.

17 London 2004, 1993.

18 Temperley bedient sich bei seinen Analysen der metrischen Struktur der graphischen Darstel- 
lungsweise der GTTM: Punkte werden sowohl horizontal, den einzelnen Zählzeiten einer Takteinheit entsprechend, als auch vertikal angeordnet. Die Anzahl vertikal dargestellter Punkte entspricht dem relativen Gewicht einer Zählzeit. Im Gegensatz zur graphischen Repräsentation der Rhythmustheorie von Cooper \& Meyer (1960), die auf eine der Prosodie entlehnte Versmaßnotation zurückgreifen, ist die Notation der GTTM aufgrund des inhärenten Zusammenhangs der Einzelzeichen in der Lage, die Beziehung der Gewichtungsverhältnisse der einzelnen Zählzeiten präziser wiederzugeben.

19 Siehe z. B. Narmour 1977.

20 Der Begriff `Phrase « wird bei Temperley als Synonym zu 'Segment verwendet und nicht in einem anspruchsvolleren Sinn wie etwa bei William Rothstein (1989, 5), der den Begriff der Phrase an das Kriterium der tonalen, zielgerichteten Bewegung rückbindet, ein Indiz für Temperleys etwas lockeren Umgang mit musiktheoretischen Termini.

21 Ein Nachteil der GTTM besteht laut Temperley darin, daß sie den zweiten Gruppierungstypus vernachlässigt hat. Dieses Defizit hat Fred Lerdahl (2001, 32-34) zu beseitigen versucht.
22 Meyer 1967.

23 Lesen und Hören stellen keine wechselseitig exklusiven Zugänge zur Musik dar, sondern beeinflussen und unterstützen sich gegenseitig. Siehe dazu Dahlhaus 1970, 62-65.

24 Temperley 1995.

25 Fodor 1983.

26 Arbitrarität ist ein Konzept, das aus der Linguistik stammt. Es besagt, daß die Assoziation zwischen einem Zeichen und dem Bezeichneten durch konventionelle Übereinkunft geregelt und damit beliebig ist. Die Assoziation zwischen Signifikant und Signifikat beruht also nicht auf den Eigenschaften der internen Struktur der beiden Relata.

27 Temperley sieht die Bedeutung musikalischer Abschnitte in deren musikinterner Funktion im Sinne der musikalischen Infrastruktur (S. 326). Die Konsequenz dieser Auffassung für den Fall der V-I-Kadenz wäre die Redeweise, daß diese Art von Kadenz Geschlossenheit sbedeutet،. Wiederum ist die Ausblendung alternativer musikalischer Bedeutungstheorien, wie sie derzeit z.B. im Bereich der Musiksemiotik vertreten werden, auffällig.

\section{Literatur}

Cook, Nicholas (1987a), »The Perception of Large-Scale Tonal Closure", Music Perception 5, 197-206.

_ (1989), »Music Theory and 'Good Comparison«: A Viennese Perspective«, Journal of Music Theory 33, 117-141.

_ (1994), „Perception: A Perspective from Music Theory«, in: Musical Perceptions, hg. von Rita Aiello, Oxford: Oxford University Press, 64-95.

(1990), Music, Imagination, and Culture, Oxford: Clarendon Press.

(1987b), A Guide to Music Analysis, London: Dent.

Dahlhaus, Carl (1970), Analyse und Werturteil, Mainz: Schott.

(1987), Beethoven und seine Zeit, Laaber: Laaber.

DeBellis, Mark (2002), „Music Analysis as Articulation", The Journal of Aesthetics and Art Criticism60, 119-135.

Fodor, Jerry A. (1983), The Modularity of Mind, Cambridge, MA: MIT Press.

Gjerdingen, Robert O. (1988), A Classical Turn of Phrase. Music and the Psychology of Convention, Philadelphia: University of Pennsylvania Press.

Huron, David (1989), Voice Segregation in Selected Polyphonic Keybord Works by Johann Sebastian Bach, Ph.D. Diss., Nottingham University. 
(2001), »Tone and Voice: A Derivation of the Rules of Voice-Leading from Perceptual Principles", Music Perception 19, 1-64.

Lerdahl, Fred \& Jackendoff, Ray (1983), A Generative Theory of Tonal Music, Cambridge, MA: MIT Press.

Lerdahl, Fred (2001), Tonal Pitch Space, Oxford: Oxford University Press.

London, Justin (2004), Hearing in Time, Oxford: Oxford University Press (1993), »Loud Rests and Other Strange Metric Phenomena, or Meter as Heard", Music Theory Online.

Meyer, Leonard B. (1967), Music, the Arts, and Ideas, Chicago: University of Chicago Press.

—_ (1973), Explaining Music, Chicago: University of Chicago Press.

Narmour, Eugene (1977), Beyond Schenkerism. The Need for Alternatives in $\mathrm{Mu}$ sic Analysis, Chicago: The University of Chicago Press.

Rothstein, William (1989), Phrase Rhythm in Tonal Music, New York: Schirmer.

Temperley, David (1995), »Motivic Perception and Modularity", Music Perception 13, 141-69.

(1996), »The Perception of Harmony and Tonality: An Algorithmic Approach«, Ph.D. Diss., Columbia University. (1997), »An Algorithm for Harmonic Analysis«, Music Perception 15, 31-68.

_ / Daniel Sleator (1999), »Modeling Meter and Harmony: A Preference Rule Approach «,ComputerMusicJournal23, 10-27. (2001a), The Cognition of Basic Musical Structures, Cambridge und London: The MIT Press. (2001b), »The Question of Purpose in Music Theory: Description, Suggestion, and Explanation«, Current musicology 66, 66-85. / Christopher Bartlette (2002), »Parallelism as a Factor in Metrical Analysis", $\mathrm{Mu}$ sic Perception 20, 117-49.

- (2004a), „Bayesian Models of Musical Structure and Cognition«, Musicae Scientiae 8, 175-205.

(2004b), „Communicative Pressure and Evolution of Musical Styles«, Music Perception 21, 313-37.

- (2005), »Review of ,Empirical Musicology: Aims, Methods, Prospectsı, ed. by Eric Clarke \& Nicholas Cook", Music Perception 23, 91-96.

Zbikowski, Lawrence (2002), Conceptualizing Music: Cognitive Structure, Theory, and Analysis, New York: Oxford University Press.

\section{Rezensionen}

Cohen, Annabel J. (2004), „D. Temperley, „The Cognition of Basic Musical Structures«", Psychology of Music 32, 105-18.

Cox, Arnie (2002), »The Cognition of Basic Musical Structures« (review)«, Quarterly Journal of the Music Library Association 59, 97-99.

Gjerdingen, Robert (2004), »)The Cognition of Basic Musical Structures by David Temperley", Music Theory Spectrum 26, 336-40. Meridith, David (2002), »Review of 'The Cognition of Basic Musical Structures` by David Temperley«, Musicae Scientiae 6, 284-302. 